\title{
A predictive model of the building design process
}

COLIN GRAY and EDWARD COLES

Department of Construction Management

University of Reading. Whiteknights, Reading, UK

In a complex construction industry, such as that in the UK, the building process is predominantly that of assembling components made off-site. This requires that all the components are detailed, together with the methods of fixing, sufficiently early to ensure that the components are delivered to site at the right time. The effect of this has been to increase the number of drawings produced by the design team, for a typical project, to between 500 and 5000. Frequently this process fails because its complexity is not understood nor managed sensibly.

The research described in the paper attempts to evaluate the hypothesis that: implicit within the technology of the project is a design task and that the tasks are linked together to give an interrelated network of the design process. An AI approach is being adopted in several ways to examine the problem. It is being used in the search for patterns of data flow to create the network and thus evaluate the hypothesis and also to build a network model of the design process.

The final model will be linked to the construction activity model TIME, also written in Prolog 2, to form a model of the complete construction procurement task.

Key words: Design, management, Prolog 2, expert systems, construction.

\section{Introduction}

In the UK building industry there is a growing awareness that the problem of the production of the design information is becoming increasingly complex and that it has to be managed in a better way than it has been upto now, (Faster Building for Industry, 1983). It is common practice that the responsibility for the content of the design is retained by the lead designer, normally the architect, who is expected to supply the information to meet the demands of the contractor. What is unusual about the practice of the UK design teams, compared to European and American practice, is that they are responsible for and active in the production of the detailed working drawings. There is, therefore, a very large overlap between the design and construction stages. However, in this approach there is no one with a clear overall view of the total process and its integrated nature with the construction sequence. When it is also considered that there can be anything between 500 and 5000 drawings on a project, then it hardly 
surprising that this process often fails to deliver the information when it is required (Davies, 1984) through failing to understand the complexity of the process (Gray, 1981).

\section{Objectives of the research}

This research project aims to develop a (computer) tool for planning and controlling the design process. While proprietry software is currently used for this purpose, for example; network analysis, such programmes require considerable forethought in defining activities and later adjustment of resource allocations. Often, a clear picture cannot be obtained until considerable design work has been done by several design disciplines in analysing an outline design proposal. In contrast the design process planner will function from a short list of the main characteristics of a sketch design and the main features of the environment in which the design is to be developed.

The proposed model will work from the deep logic of design methodology, questioning the user about the project and the management context. It will then quickly check the feasibility of the timescale and formulate a plan of action which can guide the allocation of resources.

The main outputs of the proposed model will be:

1 Logic diagrams for design decision sequences. These will be presented as 'ladder charts' which will define he content of information which is necessary to each design discipline at each stage of their work, together with the output which is required from them.

2 By interfacing the program with the existing expert system TIME for the prediction of construction activity (Gray and Little 1985) the schedule for supplying the design information can be produced. Further analysis would display the needs for: skills, personnel, facilities and cash flow, required during the detailed design process.

The hypothesis

Design and manufacture of component assemblies establishes the design model

The identification, procurement and application of knowledge domains forms the basic design process. Each component of knowledge is used to identify the specification of the materials, components and component assemblies to be use to build the final building. Therefore, once the components have been identified the linear sequence for obtaining the knowledge can be established. The sequence must recognize the ability of 
each knowledge domain to contribute to the design solution and how the specification will be established to obtain any further information it may require from the secondary domain. It has been observed on many building projects that each component and assembly carries with it a standard sequence of activities for this process. The content of the design activity will vary from project to project because the problem is varied by the conceptual design requirements.

Identification of the interfaces between the knowledge domains

If the design process for a building was a series of sequential operations functioning in parallel then the whole process would be relatively simple. However, because each component is fixed to another there is an interaction between the design process of each component to ensure that the knowledge of the fixing and support provisions is compatible from one component to another. This is particularly relevant when one component contains an element of the fixing for another component.

Design is a series of interlinked sequences between knowledge domains

The sequences and interfaces form a network of design activity using the knowledge within the domains. Each activity is established by the components being used to satisfy th requirements of the concept of the building. The components have physical attributes and can be identified, thus the network of the design activity which precedes the construction activity can be established.

This is the theory. In practice it is difficult to identify the network as design is perceived as being satisfied by two extremes. The first is that the process of aquiring knowledge is satisfied when the designer, in the corporate sense, ie, the architectural practice, is employed. The second is that the design process is satisfied when the drawings are produced. These two extremes are unsuitable for control as the first is too coarse for any method of control and the second is impossibly detailed (Farrell, 1968). An intermediate stage must be achieved to obtain a realistic assessment of the network of interchanges and to provide a level of detail on which it is possible to realistically assess progress.

\section{Data base assembly through case studies}

The hypothesis is being tested by assembling a data base of observations of the actions of designers during each stage of designing a project. According to De Marco (Yourdon, 1979), business tasks can be clearly defined in terms of their inputs and outputs. In the case of building design these are primarily:

1 task inputs of: skill, time, facilities, motivation, briefing and information. 
2 task outputs of: design decisions, drawings, calculations, documents and models

As indicators of performance, information transfer records will indicate the roles and responsibilities of the parties in the design process.

\section{Contextual variables}

The procurement method used on as project (contract structure) and the location of decision making power (organization structure) have direct influence over the boundaries of design responsibility and the transfer of design information (Walker, 1984). These primary variables in project organization interact with the internal organization, experience and motivations of the design teams to generate infinate possibilities for variation in project content. The initial attempt is to discount these factors from the data and to deal with the problem once the true design process has been revealed.

\section{The iterative loop}

Provisional decisions from one design task can be essential information on which to base design decisions for another component or system. Further, the output of subsequent design tasks may be necessary to finalise decisions for the first component or system. This interdependancy of design decisions is described as 'iteration' whereby loops seem to occur in the circulation of design information. If at each iteration the information should become more precise as opposed to traveling twice in the loop.

The classification problem

There are inadequacies in the existing classification systems for design information. It has been shown (Ashari, 1986) that both SfB and the Common Arrangement systems give unequal treatment to different phases of building projects. To overcome this a basic classification system enabling many attributes to be listed has been established with a natural language processor enabling interogation by a casual user.

The inference engine

A program has been written to examine the data from the case studies to test the hypothesis that there are patterns of design associated with each significant component set. It will be used to determine the steps in the sequence and the inputs and outputs. These links will then be used to form a complete network of the design process. As more case studies are input the sequences for similar components will be examined to build up a statistical sample to evaluate the hypothesis. So far there are more instances which support the case than not. 
The case study data base will form the basis for the design planner. A new inference program will be created to seek the systems appropriate to the particular design. From the combination of systems, the design activity cells can be identified and information transfer linkages proposed. A significant programming problem is to link activities across systems which have been inactivated in the data base structure. This is the main reason for using an AI based approach which can reason heuristically, maybe producing alternative design decision logic diagrams for evaluation either by the model's own mechanism or by the user. This work has just started.

\section{Conclusions}

The work described in this paper has of necessity had to concentrate on the problem because it is breaking new ground. Great difficulties are being experienced because design is such an emotive issue involving intense personal endeavor. However, it is anticipated that, by concentrating on the mechanistic aspects of design, a method can be proposed which does not inhibit design freedom, but allows the process to be manged to satisfy the demands of the construction process.

\section{References}

Ashari, MH (1986) Project information system, Dissertation in part fulfillment of an MSc, University of Reading.

Davies, MK (1984) The subcontractor's dilemma, CIOB Technical Information Service, No 32, London, Chartered Institute of Building.

DeMarco, T (1979) Structured analysis and system specification, Prentice Hall, New York.

Farrell, EK (1968) Programme management of power station construction, Central Electricity Generating Board, London.

Faster Building for Industry, (1983) Building EDC, London

Gray, C (1981) Management of the construction process, Building Construction and Management, London, Chartered Institute of Building.

Walker, A (1984) Project Management in Construction, Granada. 